\title{
Of Model Pets and Cancer Models: An Introduction to Mouse Models of Cancer
}

\author{
Andrea Lunardi, Caterina Nardella, John G. Clohessy, and Pier Paolo Pandolfi ${ }^{1}$ \\ Cancer Genetics Program, Beth Israel Deaconess Cancer Center, Department of Medicine and Pathology, \\ Beth Israel Deaconess Medical Center, Harvard Medical School, Boston, Massachusetts
}

\begin{abstract}
The extraordinary endeavor to faithfully model human disorders in mice began in the early 1900s, and in the century since has delivered fundamental advances in our understanding and treatment of human disease. Although it could not be appreciated at the time, $99 \%$ of mouse protein-coding genes have an equivalent homolog in the human genome, despite the striking differences in appearance between mouse and man. This remarkable genetic similarity, together with our ability to finely engineer the murine genome, has made the mouse the ideal animal in which to model and analyze human biology and disease. Here we describe this remarkable shared journey between human and mouse, and envisage the next generation of mouse models, which will no doubt prove increasingly sophisticated and even more faithful to human disease. We also address the strategic use of mice in the fight against cancer, and the role they will play in the development of therapies to eradicate this disease.
\end{abstract}

Of the many legacies bequeathed to the world by Asia, one of the most surprising is the ancient Chinese passion for "fancy mice," or mice bred as unique and invaluable pets. Written accounts from as long ago as 1100 B.C.E. describe a tradition that matured into an incredible system of breeding strategies focused on somatic traits such as the color of the coat, of the eyes, and the body size. Although China may have been the cradle of the selective breeding of these little mammals, the popular practice soon spread to the rest of the ancient world, and the interest in mouse breeding first arrived in Europe in the early part of the 17th century.

The use of mice for scientific purposes, however, would not commence for almost 200 years. Indeed, it was not until 1900 that Harvard researcher William Castle and a contemporaneous team led by Leo Loeb and mouse breeder Abbie Lathrop at the University of Pennsylvania began to study the genetic determinants responsible for the inheritance of specific somatic traits in mice and the potential correlation between the incidence of spontaneous tumors and specific mouse strains (Lathrop and Loeb 1915a,b, 1918). The idea that mice might represent useful models to disentangle the genetic forces driving human cancer susceptibility and resistance also sparked the interest of a promising undergraduate working with Castle, Clarence Cook Little. But while studying the role of the immune system in cancer transplantation, Little first encountered one of the fundamental problems in mouse modeling: the genetic heterogeneity caused by crossing different mouse strains. The extreme variability of Little's initial results, and the observation that the inheritance of somatic traits was not solely Mendelian, dictated his decision to generate pure "inbred" genetic strains by systematically crossing

\footnotetext{
${ }^{1}$ Correspondence: ppandolf@bidmc.harvard.edu

(c) 2014 Cold Spring Harbor Laboratory Press

Cite this introduction as Cold Spring Harb Protoc; doi:10.1101/pdb.top069757
} 


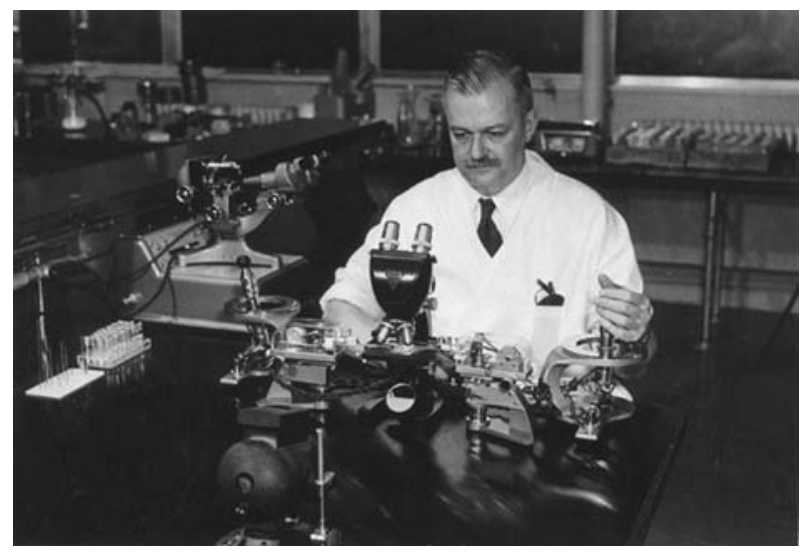

FIGURE 1. Clarence Cook Little, founding father of The Jackson Laboratory and an early pioneer in mouse genetics. (Reprinted from Nadeau 2002, by permission of Macmillan Publishers Ltd, (C) 2002.)

siblings. This approach not only reduced the variability of Little's results, but also represented a turning point in the use of mice as animal models in medical research. Little went on to author a range of pioneering studies, was the first to propose mice as faithful models of human cancer and its genetics (Castle and Little 1909, 1910; Little 1911, 1913, 1914), and eventually founded The Jackson Laboratory, a world-renowned institution for the application of genetic animal modeling to the study of human pathologies (http://jaxmice.jax.org) (Fig. 1).

\section{WHEN GENIUS MEETS TECHNOLOGY}

The transition from passive observation of spontaneous tumors in specific inbred strains to active genetic manipulation of the mouse genome began during the second half of the 20th century. In April 1974, Rudolf Jaenisch and Beatrice Mintz (Fig. 2A,B) published an account, in the Proceedings of the National Academy of Science (PNAS), of the first genetically modified mouse to carry viral DNA stably inserted into the genome of its somatic cells. Unexpectedly, however, no signs of tumor development were found in the resulting 1-yr-old mice and, because there was no integration of the SV40 DNA into the germline, the founders did not pass the transgene on to their offspring (Jaenisch and Mintz 1974). The first stable and heritable insertion of exogenous DNA into the mouse genome was obtained two years later, in 1976, when Rudolf Jaenisch infected mouse embryos at the preimplantation stage of four to eight cells with the Moloney leukemia virus (M-MuLV), creating the first transgenic mouse model of cancer (specifically leukemia) that transmitted both transgene and phenotype to the offspring following Mendelian rules (Jaenisch 1976).

Although Jaenisch and Mintz's work was essential to pave the way for murine engineering, a further technical breakthrough for the generation of stable "transgenic" mouse lines came with the development of a method to inject exogenous recombinant DNA into the pronuclei of fertilized mouse oocytes. The first publication describing the stable insertion of an exogenous DNA fragment into the mouse genome via this method appeared in Nature on November 5, 1981. Franklin Costantini and Elizabeth Lacy of Oxford University were able to integrate into the genome of (C57BL/ $6 \mathrm{xCBA} / \mathrm{H})$ fertilized eggs. Thirty percent of the resulting second generation $\left(\mathrm{F}_{2}\right)$ was characterized by the presence in their genome of the $\lambda R \beta G 2$ transgene, definitively proving the generation of the first stable engineered transgenic mouse line (Costantini and Lacy 1981). One month later, on December 11, 1981, Jon W. Gordon and Frank H. Ruddle (Yale University) published, in Science, the generation of another engineered mouse model, characterized this time by the stable genomic insertion of human interferon cDNA cloned in the pBR322 vector. pIf- 4 , the name of the founder female, was able to transmit with a Mendelian ratio the exogenous DNA to her offspring $\left(F_{1}\right)$ when crossed with a 

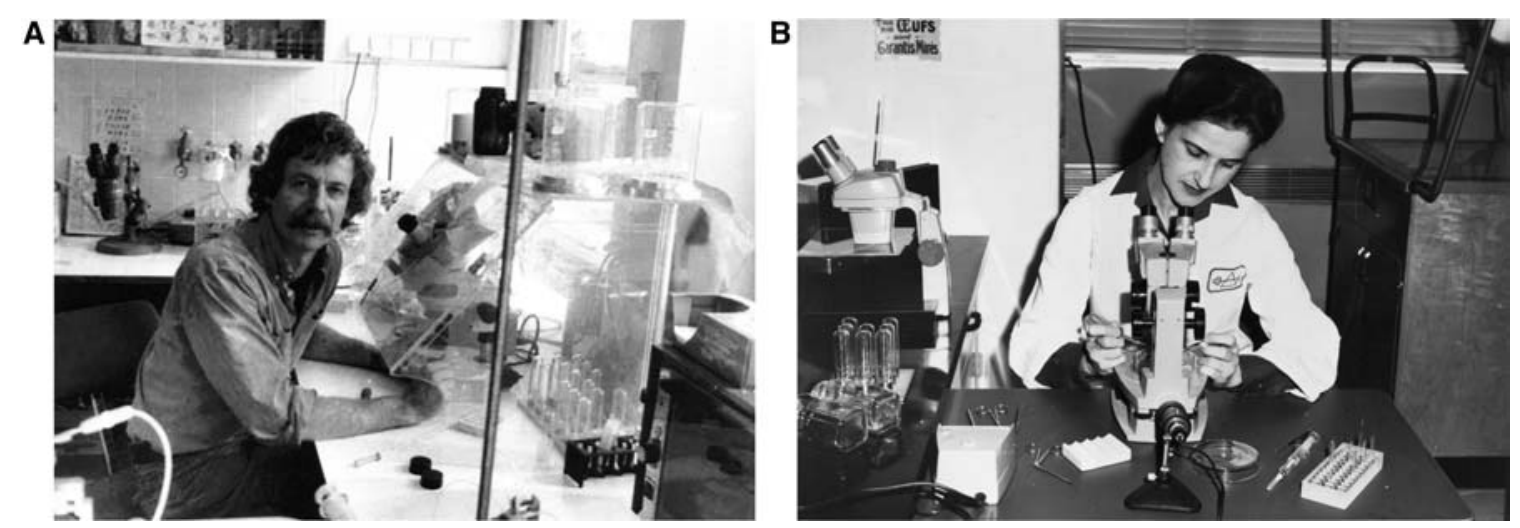

FIGURE 2. Rudolf Jaenisch $(A)$ and Beatrice Mintz $(B)$ generating the first genetically modified mouse to carry viral DNA stably inserted into the genome of its somatic cells. ( $A$, Image courtesy of R. Jaenisch; $B$, Reprinted, with permission, from Acc. 90-105-Science Service, Records, 1920s-1970s, Smithsonian Institution Archives.)

wild-type male, which served as a proof of principle for the beginning of a new era of genetic engineering and mouse modeling (Gordon and Ruddle 1981; Ruddle 1981).

Even as awareness grew that the generation of transgenic mice was now a tractable and reproducible methodology, excitement was also building in the cancer research community over the successful molecular cloning of viral and cellular oncogenes (Bishop 1985a,b,c). Inevitably, the two areas of research came together with the generation of the first series of "oncomice," transgenic mouse models overexpressing different oncogenes and characterized by the heritable predisposition to develop specific types of cancer.

The first "oncomouse" was published in Cell in June 1985 thanks to a fruitful collaboration between Richard Palmiter and Ralph Brinster (Palmiter and Brinster 1985). They were initially interested in studying the effect of rat growth hormone $(\mathrm{GH})$ overexpression in transgenic mice $(M t 1-G H)$. To counteract the low levels of the hormone and obtain a more reliable expression of the integrated transgene, Palmiter fused the entire SV40 early region, including enhancer, promoter, and large/small T-antigens coding sequence, upstream of the Mt1-GH synthetic gene (MGH) but in the opposite direction $(S V-M G H)$, with the intent being to increase the activity of the $M t 1$ promoter through the proximity of the SV40 enhancer (Palmiter and Brinster 1985).

The result of this intervention was totally unexpected. Although Rudolf Jaenisch and Beatrice Mintz had previously published that the SV40 large/small T-antigens were absolutely unable to drive cell transformation in vivo in engineered mice (Jaenisch and Mintz 1974), the SV-MGH transgenic mice of Palmiter and Brinster were clearly predisposed to develop and transmit to their offspring brain tumors from the epithelial cell layer of the choroid plexus, as well as additional sporadic tumors in other organs, thus becoming the first transgenic mouse model of cancer (Palmiter and Brinster 1985).

This breakthrough discovery, mainly due to serendipity, was immediately followed by the generation of further transgenic mice overexpressing potential oncogenes such as $v$-Src or Myc. Although the transgenic mouse for $v$-Src (Mt1-v-Src) showed no compelling evidence of tumor development, the hypothesis-driven generation of the $E \mu-M y c$ mouse model, a collaboration between Palmiter and Brinster with Jerry Adams and Suzanne Cory, was an incredible success. The overexpression of Myc in the lymphoid cell lineage, thanks to the immunoglobulin enhancer $(E \mu)$, was sufficient to drive B-cell lymphomas in the E $\mu$-Myc transgenic mice (both founder and offspring) (Adams et al. 1985), definitely proving the oncogenic essence of the Ig-Myc translocation in human malignancies. In Decem- 
A. Lunardi et al.

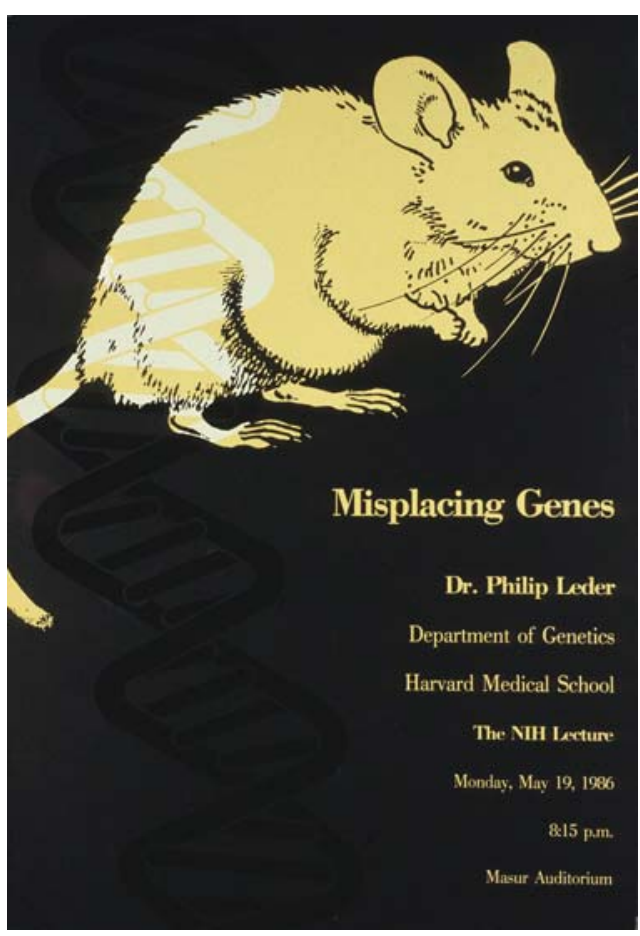

FIGURE 3. Flyer advertising Philip Leder's 1986 "NIH Lecture" on early gene targeting efforts in the mouse. The NIH Lecture series was established to facilitate interchange of information and to give appropriate recognition for outstanding scientific accomplishment. (Image reprinted from National Institutes of Health [U.S.], Medical Arts and Photography Branch, Bethesda, MD, 1986.)

ber 1985, Nature published the E $\mu$-Myc transgenic mice (Adams et al. 1985). At the same time, the team of Philip Leder and Timothy Stewart was working on $M y c$. Their attention was focused on the generation of a transgenic mouse overexpressing $M y c$ in the breast. Thanks to Leder's knowledge on the $M y c$ gene and its biology, Stewart's ability to generate transgenic mice by pronuclear injection, and the characterization of the regulatory region of a mouse retrovirus called MMTV (mouse mammary tumor virus), Stewart and Leder were able to successfully generate MMTV-Myc transgenic mice in 1983. As the two researchers hoped, mammary tumors began to appear in the transgenic females after a few rounds of pregnancy, further demonstrating the oncogenic potential of $M y c$ in this specific tissue. Published in the October 1984 (Stewart et al. 1984), the MMTV-Myc was the first transgenic mouse model of cancer to be identified by the term "oncomouse." Thereafter, the generation of mouse models for cancer research exploded in the work of many pioneering researchers, further strengthening the results obtained by Palmiter/Brinster and Leder/Stewart teams, and validating the "oncomouse" as one of the most transformational developments in the history of cancer research, with a tremendous impact on the future of this and many other scientific fields (Fig. 3).

\section{EMBRYONIC STEM CELLS AND HOMOLOGOUS RECOMBINATION: THE OTHER SIDE OF THE MOON}

However, the advent of the technology to generate transgenic mice was not the only breakthrough in mouse genetics of the 20th century. In 1981, two independent groups published a methodology for isolating and culturing in vitro mouse embryonic stem (ES) cells collected from the inner cell mass of a 3.5-d-old postcoitum mouse blastocyst (Evans and Kaufman 1981; Martin 1981). Importantly, the ES cells maintained their pluripotency in vitro and were able to generate chimeric mice when reimplanted in a new blastocyst, contributing to different cell lineages comprising the germline (Bradley et al. 1984). Within a few years, the discovery and characterization of the homologous recombination (HR) mechanisms in mammalian cells (Capecchi 1989) led to the development of techniques for the insertion of specific exogenous DNA molecules into planned genomic loci, or "gene targeting" (Capecchi 1989). 
It was in 1987 that Oliver Smithies successfully applied a gene-targeting strategy to mouse ES cells to replace a mutant form of the Hprt gene with its wild-type exogenous counterpart (Doetschman et al. 1987). The same year, Mario R. Capecchi published in Cell the generation of the first knockout (KO) mouse $\left(\mathrm{Hrpt}^{-}\right)$obtained by the illuminated functional combination of ES cell technology and an HR-based gene-targeting approach (Thomas and Capecchi 1987). The publication of the Hrpt mouse model soon after inspired the generation of a number KO mice models, which promised incredible discoveries in the fields of developmental biology and human health.

Over time, the ability to ablate a specific gene from the mouse genome, or insert a particular human disease-associated genetic mutation into a wild-type allele, became the cornerstone of a systematic effort to validate and characterize in vivo the function of genes considered oncosuppressors. Perhaps, the initial climax in these efforts was reached in 1992, when Allen Bradley's group at the Baylor College of Medicine in Houston and Robert Weinberg's group at the Whitehead Institute of the Massachusetts Institute of Technology in Boston published their $p 53$ and retinoblastoma $(R b)$ knockout mouse models in Nature (Donehower et al. 1992; Jacks et al. 1992). Both models definitively proved the fundamental oncosuppressive nature of these two genes.

The power to delete or mutate any gene of interest in the mouse genome has forever revolutionized the study of human genetic disease, and the development of these methods earned Mario R. Capecchi, Martin J. Evans, and Oliver Smithies the 2007 Nobel Prize in Physiology or Medicine.

\section{THE ONCOMOUSE PATENT}

The final decades of the 20th century would become known as an era of revolutionary changes in the landscape of biomedical research and drug development. At the same time, however, society began to consider biological discoveries as intellectual property, and hence patentable-a practice that would have a significant impact on the course of cancer research in mouse models.

In 1980 the U.S. Congress approved the Bayh-Dole Act specifically to encourage academic institutions to patent and transfer scientific innovations (developed with government funds) to industry. Many scientists, such as Palmiter and Brinster, never opted to patent their research, but three different U.S. patents were issued (in 1988, 1992, and 1999) to Harvard University based on Philip Leder and Timothy Stewart's work on the generation of Oncomice ("a transgenic non-human animal, whose germline and somatic cells contain an activated oncogene"), the derivation and use of cell lines from tumor-bearing Oncomice, and the use of Oncomice for testing carcinogenic compounds as well as anticancer drugs.

Problems quickly arose for researchers when Harvard University decided to exclusively license all three patents to the DuPont Corporation. Although the rationale for patenting scientific discoveries had been to motivate both holders and licensees to invest in further research and transformative products, the onerous fees and restrictions that DuPont imposed on sublicensees hindered the use of the Oncomice for academic research oriented toward cancer treatment. To overcome this stumbling block to research, in 2000 the National Institutes of Health (NIH) signed a series of agreements with DuPont allowing U.S. government employees and funding recipients to use Oncomice in basic biological science conducted in academic laboratories. Although of fundamental importance, this negotiation did not entirely release the research community from the broad purview of the patents and fees associated with these licenses, particularly in regard to the use of transgenic cancer-prone mouse models in the private sector and in joint projects with academia.

With the expiration of all three U.S. patents by 2007, further efforts were quickly focused on ameliorating the restrictions imposed on research use (both commercial and nonprofit) of Oncomice in drug testing, which is probably the most important commercial application of the technology. A 2005 decision by the U.S. Supreme Court strengthened the concept of "Food and Drug Administration (FDA) safe harbor," which was embedded in the Hatch-Waxman exemption (1984), and gave further protection to research in academic laboratories. The concurrent decisions of different phar- 
A. Lunardi et al.

maceutical companies to avoid licensing agreements with DuPont was further evidence of the common willingness to resolve this long-standing problem and move forward with the development of mouse models for human disease treatment optimization.

\section{ARE MICE RELEVANT MODELS FOR HUMAN DISEASE? THE ACUTE PROMYELOCYTIC LEUKEMIA (APL) PARADIGM}

Although they look very different from humans, the results of mouse (2002) and human (2003) genome sequencing have revealed that we share $>99 \%$ of our protein-coding genes with these little rodents. If this discovery still surprises the layman, it nevertheless strikingly supports the rationale for using mice as faithful model organisms in the study of genetic human diseases and possible therapies.

By now it is simply a matter of fact that mouse models have led to incredible advances in the treatment of serious and sometimes lethal pathological conditions. To select only one among a plethora of examples, the cure for a previously lethal form of hematopoietic malignancy, acute promyelocytic leukemia (APL), was optimized through experimentation on oncomice.

APL is characterized by a block in the promyelocytic stage of myeloid hemopoietic differentiation and is invariably associated with reciprocal and balanced chromosomal translocations involving chromosome 17. In 1991, several groups simultaneously identified the two genes at the breakpoint of the $\mathrm{t}(15 ; 17)$ translocation: PML on chromosome $15 \mathrm{q} 22$ and the $R A R \alpha$ gene on chromosome $17 \mathrm{q} 2$ (Longo et al. 1990; de Thé et al. 1991; Goddard et al. 1991; Kakizuka et al. 1991; Pandolfi et al. 1991). Subsequent investigations, however, revealed that APL is genetically heterogeneous and identified but six different hematologic malignancies characterized by translocations where $R A R \alpha$ is invariably involved, but its partners vary (Pandolfi 1996). This genetic diversity proved to have an impact on the way in which APL responds to treatment (Lo Coco et al. 1991).

The modeling efforts for APL proved complex, especially at that time, because of a lack of appropriate expression vectors, promoters, and regulatory elements to accurately direct in vivo, transgenic expression in the hemopoietic cellular compartments. In 1997, however, three groups lead by Mike Bishop, Timothy Ley, and Pier Paolo Pandolfi, respectively, reported the first faithful mouse models of PML-RAR $\alpha$-driven APL (Brown et al. 1997; Grisolano et al. 1997; He et al. 1997). The Pandolfi laboratory went on to model the other subtypes of APL. Critically, the generation of these models showed that clinically, molecularly, and in their response to therapy, these leukemias behaved in a similar manner to human APL. For example, PML-RAR $\alpha$ mice were found to transiently respond to retinoic acid (RA) exactly as human t(15;17) APL. As in human APL, RA was able to induce complete remission in the mouse, but leukemia would eventually relapse if treated with RA alone (Brown et al. 1997; Grisolano et al. 1997; He et al. 1997). In addition, leukemias in APL PML-RAR $\alpha$ mouse models would often become RA resistant, as does human $\mathrm{t}(15 ; 17)$ APL treated solely with RA (He et al. 1998). Furthermore, as in human $\mathrm{t}(11 ; 17)$ APL, leukemia in PLZF-RAR $\alpha$ mice was found to be resistant to RA at presentation (He et al. 1998) (Fig. 4).

Subsequently, the mouse models of APL began to inform the design of clinical trials. Results showed that leukemia in $P M L-R A R \alpha$ mice was extremely responsive to $\mathrm{As}_{2} \mathrm{O}_{3}$ (which later proved to be another powerful weapon for the treatment of APL), whereas once again leukemia in PLZF-RAR $\alpha$ mice was found to be resistant (see also below) (Lallemand-Breitenbach et al. 1999; Rego et al. 2000). By using information accrued in preclinical testing of different APL mouse models, clinicians began to stratify patients for clinical trials on the basis of genetic criteria; thus, $t(15 ; 17)$ APL patients were treated successfully with $\mathrm{As}_{2} \mathrm{O}_{3}$, whereas $\mathrm{t}(11 ; 17)$ APL patients were not (Nasr et al. 2008).

Critically, different drug combinations were tested in preclinical trials using, in a randomized fashion, the various subtypes of the APL mouse model, an effort that in a clinical setting would be lengthy and almost impossible because of the rarity of these subtypes and the prohibitive number of patients to be recruited for such screening. This preclinical approach incorporating APL mouse models proved decisive in the eradication of human APL (Lallemand-Breitenbach et al. 1999; Rego 


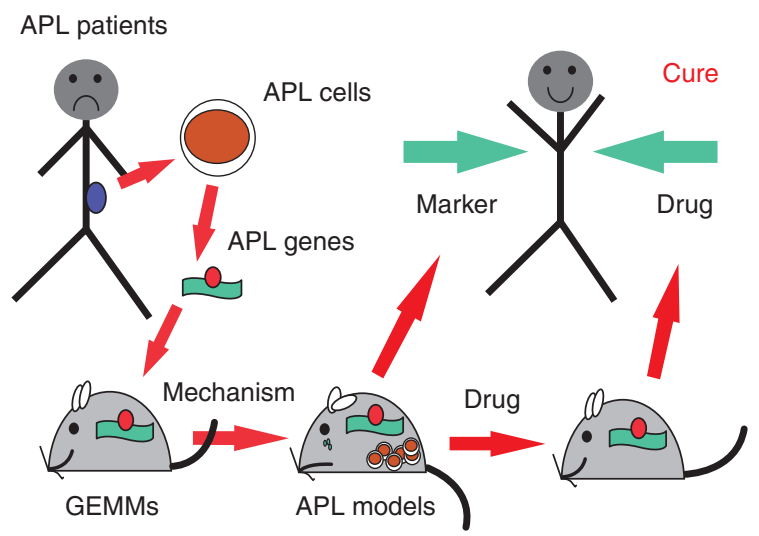

FIGURE 4. The APL journey: from genetic deconstruction toward mouse modeling to disease eradication. GEMMs, genetically engineered mouse models; APL, acute promyelocytic leukemia.

et al. 2000); as in APL RA-sensitive models, the combination of $\mathrm{As}_{2} \mathrm{O}_{3}$ and RA resulted in a cure (Nasr et al. 2008) (Fig. 4). These findings prompted the launch of a number of clinical trials with this combination in many countries worldwide, finally defining $\mathrm{As}_{2} \mathrm{O}_{3}+$ ATRA (all trans-retinoic acid) as the curative combination for RA-sensitive APL patients.

A second fundamental milestone in the APL saga was represented by the generation and use of mouse models for therapy-resistant $\mathrm{t}(11 ; 17)$ APL. To this end, Pandolfi et al. recreated the dual complexity of human $\mathrm{t}(11 ; 17)$ APL by coexpressing in transgenic animals the PLZF-RAR $\alpha$ and $R A R \alpha$-PLZF fusion proteins (He et al. 2000). These double transgenic mice developed again a classic APL-like leukemia that proved markedly resistant to both conventional chemotherapy and treatment with the $\mathrm{RA} / \mathrm{As}_{2} \mathrm{O}_{3}$ combination, as in human $\mathrm{t}(11 ; 17)$ APL (He et al. 2000). The two models were immediately enrolled in drug testing designed under a new therapeutic concept and involving a novel class of drugs: histone deacetylase inhibitors (HDACIs). The rationale for the trial was that in combination with RA, the HDACIs would overcome the transcriptional repressive activity of the PLZF-RAR $\alpha$ fusion protein (He et al. 1998, 2001). The data described by Pandolfi's group for the $t(11 ; 17)$ APL mouse model treated with RA in combination with HDACIs (SAHA) prompted the FDA to approve the testing of phenyl butyrate (a compound also active as HDACI and already approved for clinical use) in combination with RA, in therapy-resistant human APL (Warrell et al. 1998). The first patient, a 13-yr-old girl, attained complete molecular remission with negligible toxicity using a therapeutic regimen of SAHA + RA entirely derived from that optimized in mouse models of $\mathrm{t}(11 ; 17)$ APL (Warrell et al. 1998; He et al. 2001). Clinical trials with combinations of RA (ATRA) and HDACIs are still ongoing for different types of acute myeloid leukemia.

These findings represented a radical shift in the perception of mouse models of human cancer within the scientific and clinical oncology community. Clinicians and physician scientists started regarding mouse models not only as means of gaining insight into the biology of the disease, but also as powerful tools for preclinical testing of novel drugs and drug-combination therapies. These findings fostered extensive modeling efforts in other tumor types with the notion that genetically engineered mouse models (GEMMs) for other cancers would be equally beneficial in testing novel therapeutic modalities.

\section{GEMMs AND PRECLINICAL DRUG TESTING}

Today, some 30 years after the first SV-MGH model generated by Palmiter and Brinster, the technology to modify the murine genome is extremely evolved and, most importantly, widely used. Optimized breeding strategies, an incredible set of tissue and cell lineage-specific promoters able to drive transgene or recombinase (Cre) expression in a time- and compartment-dependent manner, along with the 
power to finely tune Cre activity or transgene expression through Tet- (Tet-On; Tet-Off) or estrogen receptor-inducible systems, have given rise to a wide array of GEMMs that more faithfully recapitulate most of the different types of human cancer.

Although of fundamental importance to the study of the biology of cancer, mouse models also represent by now one of the most useful in vivo platforms for drug development and the investigation of drug resistance. A key advantage of mouse models is that they allow tumors to grow in an environment characterized by a normal immune system, in the organ of origin, and surrounded by the correct stroma, all conditions that strongly affect tumor progression and response to treatment. Furthermore, tumor response to specific therapeutic regimens can be monitored by using exactly the same procedures as would be administered to human patients, such as palpation, serological measurement of tumor markers, magnetic resonance imaging (MRI), positron emission tomography (PET), computed tomography (CT)-PET, or bioluminescence, and the response classified by using the same criteria (e.g., RECIST). Finally, mice are relatively inexpensive, treatments can be easily run on a large number of homogeneous animals (genotype, age, background, sex), responses are faster than in human patients, and genetic and molecular analyses to define mechanisms of de novo or acquired drug resistance are always applicable.

Accordingly, in the last decade, GEMMs have been enrolled in a wide variety of preclinical studies with such differing goals as the following.

- Target validation. GEMMs are often used to determine whether the potential target of a new compound is indeed a key factor for tumor cell survival, proliferation, or migration (Martin et al. 2005; Toogood et al. 2005).

- Testing new and more sophisticated drugs for target therapy. GEMMs are routinely enrolled to assess tumor response to new experimental therapeutics directed against specific targets known to be deregulated in specific types of tumors. These therapeutics have included (among others) retinoic acid, tyrosine kinase receptor inhibitors, rapamycin analogs, PARP inhibitors, cyclin-dependent kinase inhibitors, PI3K/mTOR/AKT inhibitors, neoangiogenesis inhibitors, farnesyl transferase inhibitors, prostaglandin inhibitors, androgen receptor inhibitors, and DNA methyltransferase inhibitors. These, to name a few, and multiple other compounds have been tested in prevention or treatment settings in different types of mouse models (Politi and Pao 2011; Sharpless and Depinho 2006).

- Mechanisms of de novo or acquired tumor resistance. Unfortunately, to date no drug has the power to cure a cancer as a single agent. Furthermore, the most effective treatments normally show different magnitudes of response in different patients, and invariably the tumor in question will acquire some resistance to treatment. GEMMs represent an invaluable tool to quickly anticipate (years in advance of comparable studies in human patients) the duration of tumor response and the mechanisms of acquired resistance when it kicks in. Importantly, efforts to characterize the molecular mechanisms and pathways responsible for acquired resistance to specific treatment have proven GEMMs to be faithful and essential to unraveling these mechanisms. Numerous examples of such contributions by GEMMs exist, and include, among many other examples, the fact that RA treatment is ineffective in $\mathrm{t}(11 ; 17)$ APL (He et al. 2000, 2001), that chemotherapy cytotoxicity is tightly linked to Trp53 status (Lowe et al. 1993a,b), that sensitivity to EGFR inhibition can be bypassed by RAS or PI3K hyperactivity (Mellinghoff et al. 2005), that KRAS/TP53 and KRAS/LKB1 double-mutant lung cancers are resistant to docetaxel (Chen et al. 2012), and that androgen deprivation therapy is ineffective in a context where XAF1/XIAP and SRD5A1 genes are deregulated (Lunardi et al. 2013).

- Combinational therapy. Finally, GEMMs are a perfect investigational platform to test drug combinations focused to target-specific pathways identified as candidate mechanisms for tumor resistance to particular treatments. As previously described, a paramount example of the prominence of using GEMMs in this preclinical arm is represented by the discovery, initially in mouse models and then successfully translated to human patients, of the curative effect of the RA and $\mathrm{As}_{2} \mathrm{O}_{3}$ combination for the $\mathrm{t}(15 ; 17)$ APL (Rego et al. 2000; Nasr et al. 2008). 
Accordingly, in recent years different groups worldwide have poured an exponentially increasing amount of effort in this direction, with important results now covering many different types of tumors. Combination treatment with VEGFR plus bFGF inhibitors, for instance, has been effective in a mouse model of pancreatic islet carcinogenesis resistant to VEGFR inhibition alone (Casanovas et al. 2005). Other research has shown that PARP inhibition sensitizes BRCA1-null p53het mouse model of breast cancer to PI3K inhibition (Juvekar et al. 2012); AKT/mTOR and ERK/ MAPK double targeting inhibits tumor growth in the castration-resistant Pten/Nkx3.1 mutant mouse model of prostate cancer (Kinkade et al. 2008; Floc'h et al. 2012); XIAP and SRD5A1 double inhibition through Embelin plus Dutasteride treatment rescues ADT efficacy in castration-resistant Pten/Lrf and Pten/p53 double null prostate cancers mouse models (Lunardi et al. 2013); and MEK inhibitor selumetinib provides substantial benefit to Kras/Trp53 but not to Kras/ Lkb1 double null lung cancer mouse models resistant to docetaxel as monotherapy (Chen et al. 2012).

Although these studies represent only a few of the many published examples, they are, we hope, plentiful to convey the clinical relevance of GEMMs to optimize the treatment of human genetic diseases such as cancer.

\section{XENOGRAFTS, ALLOGRAFTS, AND CARCINOGEN-INDUCED TUMORS}

Transplantation models and chemical inducers have been used routinely for decades in cancer research. These technologies are in continuous development and, despite their well-known limitations, represent additional important tools for cancer biology and drug screening studies.

The term xenograft, from the Greek xenos plus graphion, indicates a graft of tissue obtained from a member of one species and transplanted into a member of another species. The most common xenograft approach used in cancer research laboratories is the injection of human tumor cell lines mixed with Matrigel under the skin of immunocompromised mice (i.e., nude, SCID, NOG, NSG, or $\mathrm{NOD} / \mathrm{SCID} / \gamma)$. Injected cells normally engraft, and in few weeks a solid tumoral mass is palpable. Although this method is extremely fast and reproducible, they use tumor cell lines that have been growing in vitro for decades (HeLa cell line was generated in 1951, HEK293 in early 1970, PC3 in 1979), and so carry an unknown number of mutations, genomic aberrations, and metabolic dysfunctions, which has sometimes led to complications in the correct interpretation of positive as well as negative results.

A more complex, in general less efficient, but absolutely valid alternative is the transplantation of part of a primary human tumor into mice. This kind of procedure yields the best results when the tumor is transplanted into the same organ of the host as where it arose in the patient, thus assuring a comparable growth environment. The mice commonly enrolled in xenograft transplantation studies, however, are often moderately to severely immunocompromised; although this condition is necessary to permit the transplant, the engraftment can be considered extremely artifactual and observations derived from it therefore can potentially be misleading.

Similar in concept but different in execution, transplanted tumor cells or primary tumors derived from another mouse (instead of from a human being) are known as allografts (alias syngeneic models). Accordingly, transplants can be done in immune-competent wild-type host mice without any risk of rejection, because they do not suffer from distinct immune responses, a condition that more closely mimics the classical tumor's microenvironment.

Last, but hardly of minor relevance, is the use of carcinogens to induce spontaneous tumors in mice. Starting in the 1940s with the use of mice to study the tumoral effects of polycyclic aromatic hydrocarbons on the digestive organ, today the list of tumor types induced in murine models includes breast cancer through the MMTV virus, oral and lung cancers by tobacco-derived compounds, skin cancer through the irradiation with ultraviolet light or the administration of TPA/DMBA, specific types of leukemia by ionizing radiation, and multiple types of malignancies through cadmium and asbestos. Although these models may be considered faithful platforms for selected cancer biology 
A. Lunardi et al.

studies and drug testing, generally they suffer from the same limitations as primary tumors in humans, which they may or may not mirror.

THE NEW AGE: THE CO-CLINICAL TRIAL PROJECT

In the last decade, tremendous advances in technology have allowed scientists to gain powerful insight into the molecular and genetic determinants that drive cancer. The access to technologies such as whole exome sequencing and their diminishing cost are providing new challenges to cancer care providers. With molecular pathology increasingly impacting patient care protocols, the ability to identify and evaluate novel drugs and drug combinations in molecularly categorized cancers using a randomized trial approach becomes increasingly challenging. This shift in how we believe about cancer, not only from a pathological subtype but now also by molecular subtype, heralds a new era for the mouse in cancer biology, presenting the opportunity to play a key role in redefining the paradigm by which we develop treatments and therapies for cancer patients.

Furthermore, the advancement of new engineering technologies now makes possible the generation of increasingly sophisticated and faithful mouse models that recapitulate the complex genetic events characterizing human cancer. However, although a "GEMM to human" or "preclinical to clinical" approach has already proven to be hugely effective toward the eradication of a subset of diseases (e.g., acute promyelocytic leukemia [APL]) where the genetic defects are well defined and "druggable" targets have been identified, the rate at which all this advanced knowledge is translated into effective therapeutics is still pitifully slow.

To fill this void, in 2007 Pier Paolo Pandolfi designed with Lewis C. Cantley the "Co-Clinical Trial Project" and implemented it at the Beth Israel Deaconess Cancer Center of Harvard Medical School under the auspices of the National Cancer Institute (Nardella et al. 2011).

The Co-Clinical Trial Project stems from a paradigmatic realization regarding the power of testing new experimental therapeutics and traditional standard-of-care drugs, as single agents or in combination, in faithful GEMMs, according to the simple yet transformative principle of conducting the treatments in parallel, and with exactly the same protocols as current clinical trials in human patients (Nardella et al. 2011) (Fig. 5).

The keys to this concept are "synchronicity" and "integration." More specifically, by treating GEMMs and human patients in exactly the same way, it will be possible to accrue relevant clinical, biological, and pharmacologic data (i.e., sensitivity vs. resistance to specific regimens, imaging analysis, microarray and proteomic profiling, somatic mutation, nucleotide polymorphisms, etc.) from both sources, analyze the data in parallel, integrate the information obtained, and, finally, identify biomarkers that can help to genetically or molecularly stratify patients by predicting positive or negative responses to the treatment (Nardella et al. 2011) (Fig. 6). Importantly, these efforts will uncover new mechanisms from which to built new experimental therapeutic modalities.

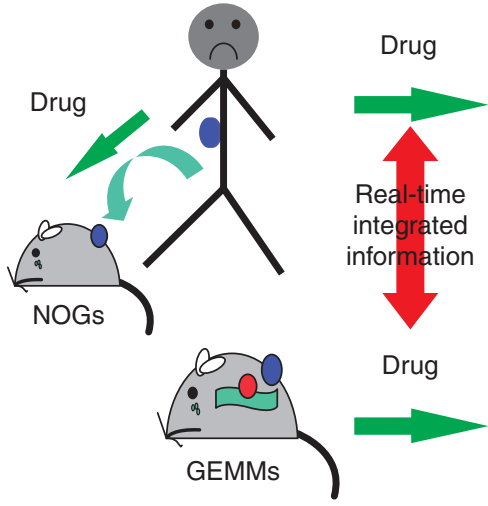

FIGURE 5. A new working paradigm: the "Co-Clinical Trial Project." Real-time integrated analysis of treatments in human patients with corresponding treatments in mouse models that faithfully model human disease can enable more efficient and effective stratification for patient treatment and result in successful clinical outcomes. 


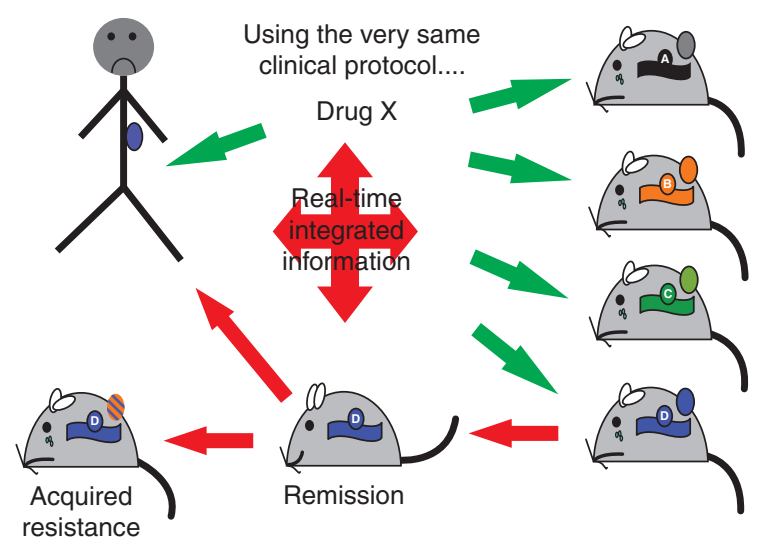

FIGURE 6. "The Co-Clinical Trial Project" beyond the preclinical (mouse) to clinical (man) dogma. A coclinical approach to the development of personalized patient care will take advantage of multiple GEM-S of human disease to identify in real time an appropriate course of treatment for successful eradication of disease.

Two outstanding examples regarding the feasibility and the power of the "Co-Clinical" approach have come from the laboratories of Pandolfi and Wong on prostate (Lunardi et al. 2013) and lung cancer (Chen et al. 2012), respectively.

The mainstay of systemic therapy for advanced prostate cancer $(\mathrm{CaP})$ for the last 70 years has been androgen deprivation therapy (ADT). This form of therapy is generally effective, with an initial success rate of almost $90 \%$. However, almost invariably the disease becomes resistant to the treatment. Interestingly, a wide variability in the endurance of ADT is well documented: Response to ADT can last anywhere from a few months to more than a decade, but little is known about the genetic and molecular determinants of the inevitable resistance that develops.

Given the variability of genetic patterns of $\mathrm{CaP}$, it has been hypothesized that the genetic forces driving progression of the disease would also affect the response to therapy.

By studying in parallel a wide cohort of human prostate cancer patients with a panel of different castration-resistant GEMMs characterized by common genetic lesions identified in human CaP, Pandolfi's group showed that this is the case (Lunardi et al. 2013). Indeed, they observed that different genetic alterations dictate a differential response to ADT in both GEMMs and human patients. Additionally, Pandolfi et al. were able to identify XAF1/XIAP- and SRD5A1-specific pathways as mainly deregulated in genetically and molecularly stratified mouse and human prostate tumors. Importantly, both pathways were found to be "druggable," and in vitro studies of human CaP cell lines as well as in vivo treatment of castration-resistant prostate cancer (CRPC) GEMMs showed a strong efficacy of Embelin (natural XIAP inhibitor) and Dutasteride (SRD5A1 inhibitor) when combined with ADT, clearly suggesting that such a drug regimen could benefit CRPC patients (Lunardi et al. 2013).

Thus, this "Co-Clinical" strategy quickly identified predictive genetic biomarkers and novel mechanisms of resistance to therapy that, if further validated in perspective clinical trial, will serve as an additional proof of principle regarding the power of this approach to facilitate their real-time identification and potentially redirect therapy-resistant patients to more effective types of treatment.

The second and very recent example of a successful application of a "Co-Clinical" strategy was the result of a collaboration focused on lung cancer between the laboratory of Kwok Kin Wong and many other investigators, including Jeffrey Engelman, Pier Paolo Pandolfi, and Lewis Cantley (Chen et al. 2012).

Lung cancer is the leading cause of cancer death in the United States and lung cancer patients are generally stratified according to a single "oncogenic states," such as mutant KRAS, LKB1, or TP53; yet cancers harboring an identical genetic aberration may be characterized by sizable differences in their response to a specific targeted therapy. As in the case of $\mathrm{CaP}$, the genetic and molecular mechanisms underlying this heterogeneous response are not fully understood.

In an attempt to gain new insight into this vexing question, Dr. Wong et al. enrolled Kras, Kras/ Trp53, and Kras/Lkbl GEMMs in a "Co-Clinical" trial mirroring an ongoing clinical trial of a combination of the MEK inhibitor selumetinib (AZD6244) with docetaxel in patients with 
KRAS-mutant lung cancer. Their studies showed that concomitant loss of either p53 or Lkb1 was sufficient to transform docetaxel-sensitive Kras-mutant cancers into resistant tumors. Importantly, this study showed that Kras and Kras/p53 lung cancer GEMMs were sensitive to the combination of selumetinib plus docetaxel, whereas the Kras/Lkb1 mutant mice showed de novo resistance to the therapy. Critically, these data were predictive of what was observed in human lung cancer (Chen et al. 2012).

Thus, these initial coclinical efforts already show significant promise for the coclinical platform to be a powerful approach toward a real-time development and optimization of patient stratification in the successful treatment of cancer.

\section{BUILDING THE MOUSE HOSPITAL}

Although GEMMs are playing a fundamental role in unraveling the cornerstone genetic mechanisms of many different human diseases, their use is still imperfect. To improve the applicability of GEMMs to help us better defeat scourges such as cancer we need to (i) define in greater detail the molecular genetic events that underlie cancer (and additionally, in those cases where the resistance to a particular treatment may be a secondary event that is completely unrelated to the initial genetic lesions), which is to say that we have to enroll more and better patients in our mouse hospital; (ii) develop standard operating procedures (SOPs) to standardize how experiments are performed and analyzed across multiple research centers; and (iii) generate an infrastructure to allow for preclinical trials in GEMMs to be performed and integrated in real time with human clinical trial data, for rapid translation of results to the clinic in a coclinical fashion.

In line with these critical needs, Pier Paolo Pandolfi with Lewis Cantley designed and launched the first "Mouse Hospital" at the BIDMC Cancer Center and Harvard Medical School, with the support of NCI in 2009 (Nardella et al. 2011). Other "Mouse Hospitals" are now operational at the NIH, Columbia University, Cold Spring Harbor Laboratory, Jackson Laboratory, and in Europe.

The Mouse Hospital represents an ideal realization of the multidisciplinary environment required for the centralized execution of drug testing in mice and its subsequent integration with data from human patients (i.e., the "Co-Clinical" approach described above).

Some of the pivotal components of the Mouse Hospital are (i) a state-of-the-art vivarium with a staff specialized in mouse husbandry, treatment, and veterinary care; (ii) a mouse pharmacy where compounds used in the trials in mice are synthesized, thereby bypassing the complexity of the material transfer agreement (MTA) process with pharmaceutical companies; (iii) in vivo imaging platforms with protocols that align with those performed in human patients both in terms of data acquisition and in terms of analysis; (iv) a center for biomarkers and comparative pathology, where human/mouse markers are evaluated for the purposes of diagnosis and prognosis, and that includes the development of tissue-based assays aimed at identifying, validating, and assessing these biomarkers in cancers and in response to treatments; (v) a bioinformatics data analysis center that assures open access to bioinformatics tools for integrated analysis of data as well as toxicity, along with IC50 and protocols for drug administration, with the ultimate goal of generating databases for genetically stratified human and mouse "patient" samples and their response to treatments; and (vi) a center for the development of new and improved mouse models that faithfully recapitulate the genetics of disease.

Thus, the "Mouse Hospital" represent an extension of the clinical cancer center, whose successful integration requires strong interaction between clinical experts and scientific staff to ensure that trials in mice are designed in a manner that faithfully mirrors human trials for the benefit of the patients, which can subsequently inform clinicians regarding patient stratification and allow for implementation of personalized treatment protocols. It is this collaboration among geneticists, biologists, bioinformatics, chemists, pharmacologists, radiologists, pathologists, and clinicians that lies at the core of the mouse hospital and represents a necessary prerequisite for the valid application of the coclinical and postclinical platforms (Nardella et al. 2011; Chen et al. 2012; Lunardi et al. 2013) (Fig. 7). 


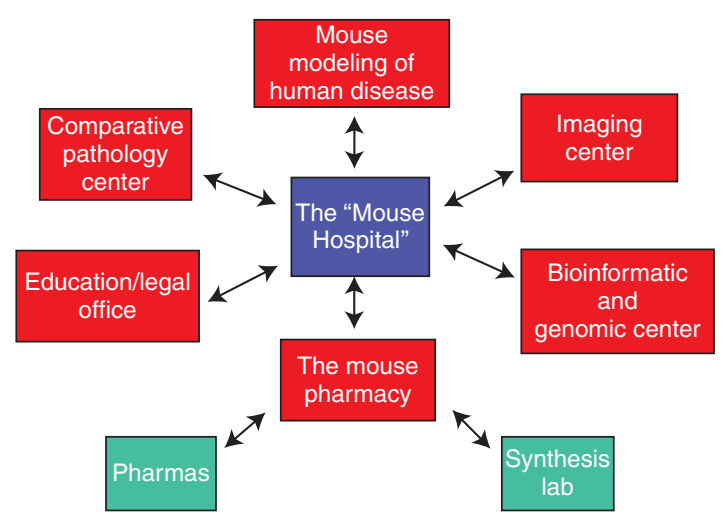

FIGURE 7. Classification of the various strategic approaches that fall under the "Co-Clinical" paradigm.

It is what we owe to patients, who still daily succumb to this horrible disease. We simply cannot fail them.

\section{CONCLUSIONS}

In the past two decades the rising improvement of sequencing technologies has enabled the attainment of an incredible amount of molecular and genetic information regarding the mechanisms driving human diseases, primarily cancer, and in doing so is changing the face of how we think of cancer and manage patient care. Concurrent advances in genetic engineering have likewise provided us the tools to generate GEMMs that finely mirror many of the genetic aberrations characterizing human diseases. The sophisticated mouse models generated over the past few years have contributed tremendously to disentangling the basic mechanisms responsible for various tumor types, neural and immune disorders, diabetes, and other diseases and have allowed us to screen the efficacy of new compounds in thousands of preclinical studies along the way. However, positive results obtained in preclinical studies using mouse models have not always translated to successful clinical trials in patients. Accordingly, the generation of ever more faithful, robust, and cost-effective mouse models, together with the realization of centralized "Mouse Hospitals" able to conduct large-scale mouse trials by using preclinical, coclinical, or postclinical platforms, are necessary to further improve the predictive potential of GEMMs in clinical studies (Nardella et al. 2011; Chen et al. 2012; Lunardi et al. 2013) (Fig. 7).

Although the use of GEMMs and high-tech in vivo analysis may be considered expensive, and obtaining useful quantities of investigational drugs from pharmaceutical companies remains challenging, the costs are minimal when compared with the millions of dollars wasted on failed human clinical trials. Importantly, the coclinical approach may represent a real breakthrough in the effort to quickly stratify responsive patients from resistant patients enrolled in phase I/II clinical trials, on the basis of precise biomarkers. Simultaneously, this approach may allow us to identify the molecular mechanisms dictating drug resistance. Such analysis will accelerate the process for approval of new drugs, diminish clinical trial failure, and serve as a rationale for quickly enrolling resistant patients in new combination treatments based on the molecular diagnosis of their disease.

\section{ACKNOWLEDGMENTS}

We dedicate this to all the people who along the way gave their contributions, famous or unknown, to the development of mouse genetic engineering, one of the most important and effective instruments for the study of human diseases. 
We thank Cheryl Marks, Dinah Singer, and Lewis C. Cantley for the fundamental critical discussions that paved the way for the realization of the Co-Clinical approach. A special thanks to Thomas Garvey for insightful editing. The Co-Clinical paradigm whose principles and preliminary important results are described here has been supported by the Mission Hope Cancer Center/National Cancer Institute (MHCC/NCI) through the RC2 (CA147940-01) and the Leadership (CA141457-04) Grants to P.P.P. A.L. has been supported in part by a fellowship from the Istituto Toscano Tumori (ITT, Italy).

\section{REFERENCES}

Adams JM, Harris AW, Pinkert CA, Corcoran LM, Alexander WS, Cory S, Palmiter RD, Brinster RL. 1985. The c-myc oncogene driven by immunoglobulin enhancers induces lymphoid malignancy in transgenic mice. Nature 318: 533-538.

Bishop JM. 1985a. Exploring carcinogenesis with retroviral and cellular oncogenes. Progress Med Virol 32: 5-14.

Bishop JM. 1985b. Viral oncogenes. Cell 42: 23-38.

Bishop JM. 1985c. Viruses, genes, and cancer. II. Retroviruses and cancer genes. Cancer 55: 2329-2333.

Bradley A, Evans M, Kaufman MH, Robertson E. 1984. Formation of germline chimaeras from embryo-derived teratocarcinoma cell lines. Nature 309: 255-256.

Brown D, Kogan S, Lagasse E, Weissman I, Alcalay M, Pelicci PG, Atwater S, Bishop JM. 1997. A PMLRAR $\alpha$ transgene initiates murine acute promyelocytic leukemia. Proc Natl Acad Sci 94: 2551-2556.

Capecchi MR. 1989. The new mouse genetics: Altering the genome by gene targeting. Trends Genetics 5: 70-76.

no Casanovas O, Hicklin DJ, Bergers G, Hanahan D. 2005. Drug resistance by evasion of antiangiogenic targeting of VEGF signaling in late-stage pancreatic islet tumors. Cancer Cell 8: 299-309.

Castle WE, Little CC. 1909. The peculiar inheritance of pink eyes among colored mice. Science 30: 313-314.

Castle WE, Little CC. 1910. On a modified Mendelian ratio among yellow mice. Science 32: 868-870.

Chen Z, Cheng K, Walton Z, Wang Y, Ebi H, Shimamura T, Liu Y, Tupper T, Ouyang J, Li J, et al. 2012. A murine lung cancer co-clinical trial identifies genetic modifiers of therapeutic response. Nature 483: 613-617.

Costantini F, Lacy E. 1981. Introduction of a rabbit $\beta$-globin gene into the mouse germ line. Nature 294: 92-94.

de Thé H, Lavau C, Marchio A, Chomienne C, Degos L, Dejean A. 1991. The PML-RAR $\alpha$ fusion mRNA generated by the $t(15 ; 17)$ translocation in acute promyelocytic leukemia encodes a functionally altered RAR. Cell 66: 675-684.

Doetschman T, Gregg RG, Maeda N, Hooper ML, Melton DW, Thompson S, Smithies O. 1987. Targetted correction of a mutant HPRT gene in mouse embryonic stem cells. Nature 330: 576-578.

Donehower LA, Harvey M, Slagle BL, McArthur MJ, Montgomery CA Jr, Butel JS, Bradley A. 1992. Mice deficient for p53 are developmentally normal but susceptible to spontaneous tumours. Nature 356: 215-221.

Evans MJ, Kaufman MH. 1981. Establishment in culture of pluripotential cells from mouse embryos. Nature 292: 154-156.

Floc'h N, Kinkade CW, Kobayashi T, Aytes A, Lefebvre C, Mitrofanova A, Cardiff RD, Califano A, Shen MM, Abate-Shen C. 2012. Dual targeting of the Akt/mTOR signaling pathway inhibits castration-resistant prostate cancer in a genetically engineered mouse model. Cancer Res 72: 4483-4493.

Goddard AD, Borrow J, Freemont PS, Solomon E. 1991. Characterization of a zinc finger gene disrupted by the $\mathrm{t}(15 ; 17)$ in acute promyelocytic leukemia. Science 254: 1371-1374.

Gordon JW, Ruddle FH. 1981. Integration and stable germ line transmission of genes injected into mouse pronuclei. Science 214: 1244-1246.

Grisolano JL, Wesselschmidt RL, Pelicci PG, Ley TJ. 1997. Altered myeloid development and acute leukemia in transgenic mice expressing PMLRAR $\alpha$ under control of cathepsin G regulatory sequences. Blood 89: 376-387.

He LZ, Tribioli C, Rivi R, Peruzzi D, Pelicci PG, Soares V, Cattoretti G, Pandolfi PP. 1997. Acute leukemia with promyelocytic features in PML/ RAR $\alpha$ transgenic mice. Proc Natl Acad Sci 94: 5302-5307.
He LZ, Guidez F, Tribioli C, Peruzzi D, Ruthardt M, Zelent A, Pandolfi PP. 1998. Distinct interactions of PML-RAR $\alpha$ and PLZF-RAR $\alpha$ with corepressors determine differential responses to RA in APL. Nat Genetics 18: $126-135$.

He LZ, Bhaumik M, Tribioli C, Rego EM, Ivins S, Zelent A, Pandolfi PP. 2000. Two critical hits for promyelocytic leukemia. Mol Cell 6: 11311141.

He LZ, Tolentino T, Grayson P, Zhong S, Warrell RP Jr, Rifkind RA, Marks PA, Richon VM, Pandolfi PP. 2001. Histone deacetylase inhibitors induce remission in transgenic models of therapy-resistant acute promyelocytic leukemia. J Clin Invest 108: 1321-1330.

Jacks T, Fazeli A, Schmitt EM, Bronson RT, Goodell MA, Weinberg RA. 1992. Effects of an Rb mutation in the mouse. Nature 359: 295-300.

Jaenisch R. 1976. Germ line integration and Mendelian transmission of the exogenous Moloney leukemia virus. Proc Natl Acad Sci 73: 1260-1264.

Jaenisch R, Mintz B. 1974. Simian virus 40 DNA sequences in DNA of healthy adult mice derived from preimplantation blastocysts injected with viral DNA. Proc Natl Acad Sci 71: 1250-1254.

Juvekar A, Burga LN, Hu H, Lunsford EP, Ibrahim YH, Balmana J, Rajendran A, Papa A, Spencer K, Lyssiotis CA, et al. 2012. Combining a PI3K inhibitor with a PARP inhibitor provides an effective therapy for BRCA1-related breast cancer. Cancer Discovery 2: 1048-1063.

Kakizuka A, Miller WH Jr, Umesono K, Warrell RP Jr, Frankel SR, Murty VV, Dmitrovsky E, Evans RM. 1991. Chromosomal translocation t$(15 ; 17)$ in human acute promyelocytic leukemia fuses RAR $\alpha$ with a novel putative transcription factor, PML. Cell 66: 663-674.

Kinkade CW, Castillo-Martin M, Puzio-Kuter A, Yan J, Foster TH, Gao H, Sun Y, Ouyang X, Gerald WL, Cordon-Cardo C, et al. 2008. Targeting AKT/mTOR and ERK MAPK signaling inhibits hormone-refractory prostate cancer in a preclinical mouse model. J Clin Invest 118: 30513064.

Lallemand-Breitenbach V, Guillemin MC, Janin A, Daniel MT, Degos L, Kogan SC, Bishop JM, de Thé H. 1999. Retinoic acid and arsenic synergize to eradicate leukemic cells in a mouse model of acute promyelocytic leukemia. J Exp Med 189: 1043-1052.

Lathrop AE, Loeb L. 1915a. Further investigations on the origin of tumors in mice: I. Tumor incidence and tumor age in various strains of mice. $J$ Exp Med 22: 646-673.

Lathrop AE, Loeb L. 1915b. Further investigations on the origin of tumors in mice: II. Tumor incidence and tumor age in hybrids. J Exp Med 22: 713-731.

Lathrop AE, Loeb L. 1918. Further investigations on the origin of tumors in mice: V. The tumor rate in hybrid strains. J Exp Med 28: 475-500.

Little CC. 1911. The "Dilute" forms of yellow mice. Science 33: 896-897.

Little CC. 1913. "Yellow" and "Agouti" factors in mice. Science 38: 205.

Little CC. 1914. A possible Mendelian explanation for a type of inheritance apparently non-Mendelian in nature. Science 40: 904-906.

Lo Coco F, Avvisati G, Diverio D, Biondi A, Pandolfi PP, Alcalay M, De Rossi G, Petti MC, Cantu-Rajnoldi A, Pasqualetti D, et al. 1991. Rearrangements of the RAR- $\alpha$ gene in acute promyelocytic leukaemia: Correlations with morphology and immunophenotype. Br J Haematol 78: 494-499.

Longo L, Pandolfi PP, Biondi A, Rambaldi A, Mencarelli A, Lo Coco F, Diverio D, Pegoraro L, Avanzi G, Tabilio A, et al. 1990. Rearrangements and aberrant expression of the retinoic acid receptor $\alpha$ gene in acute promyelocytic leukemias. J Exp Med 172: 1571-1575.

Lowe SW, Ruley HE, Jacks T, Housman DE. 1993a. p53-dependent apoptosis modulates the cytotoxicity of anticancer agents. Cell 74: 957-967. 
Lowe SW, Schmitt EM, Smith SW, Osborne BA, Jacks T. 1993b. p53 is required for radiation-induced apoptosis in mouse thymocytes. Nature 362: 847-849.

Lunardi A, Ala U, Epping MT, Salmena L, Webster KA, Wang G, Mazzucchelli R, Stack E, Lis R, Patnaik A, et al. 2013. A co-clinical approach identifies mechanisms and potential therapies for androgen deprivation resistance in prostate cancer. Nat Genet 45: 747-755.

Martin GR. 1981. Isolation of a pluripotent cell line from early mouse embryos cultured in medium conditioned by teratocarcinoma stem cells. Proc Natl Acad Sci 78: 7634-7638.

Martin A, Odajima J, Hunt SL, Dubus P, Ortega S, Malumbres M, Barbacid M. 2005. Cdk2 is dispensable for cell cycle inhibition and tumor suppression mediated by p2 $7^{\mathrm{Kip} 1}$ and p21 ${ }^{\mathrm{Cip} 1}$. Cancer Cell 7: 591-598.

Mellinghoff IK, Wang MY, Vivanco I, Haas-Kogan DA, Zhu S, Dia EQ, Lu KV, Yoshimoto K, Huang JH, Chute DJ, et al. 2005. Molecular deter minants of the response of glioblastomas to EGFR kinase inhibitors. $N$ Engl J Med 353: 2012-2024.

Nadeau JH. 2002. Single-nucleotide polymorphisms: Tackling complexity. Nature 420: 517-518.

Nardella C, Lunardi A, Patnaik A, Cantley LC, Pandolfi PP. 2011. The APL paradigm and the "co-clinical trial" project. Cancer Discovery 1: 108-116.

Nasr R, Guillemin MC, Ferhi O, Soilihi H, Peres L, Berthier C, Rousselot P, Robledo-Sarmiento M, Lallemand-Breitenbach V, Gourmel B, et al. 2008. Eradication of acute promyelocytic leukemia-initiating cells through PML-RARA degradation. Nat Med 14: 1333-1342.

Palmiter RD, Brinster RL. 1985. Transgenic mice. Cell 41: 343-345.

Pandolfi PP. 1996. PML, PLZF and NPM genes in the molecular pathogenesis of acute promyelocytic leukemia. Haematologica 81: 472-482.
Pandolfi PP, Grignani F, Alcalay M, Mencarelli A, Biondi A, LoCoco F, Grignani F, Pelicci PG. 1991. Structure and origin of the acute promyelocytic leukemia myl/RAR $\alpha \mathrm{cDNA}$ and characterization of its retinoid-binding and transactivation properties. Oncogene 6: 1285-1292.

Politi K, Pao W. 2011. How genetically engineered mouse tumor models provide insights into human cancers. J Clin Oncol 29: 2273-2281.

Rego EM, He LZ, Warrell RP Jr, Wang ZG, Pandolfi PP. 2000. Retinoic acid (RA) and As2O3 treatment in transgenic models of acute promyelocytic leukemia (APL) unravel the distinct nature of the leukemogenic process induced by the PML-RAR $\alpha$ and PLZF-RAR $\alpha$ oncoproteins. Proc Natl Acad Sci 97: 10173-10178.

Ruddle FH. 1981. A new era in mammalian gene mapping: Somatic cell genetics and recombinant DNA methodologies. Nature 294: 115-120.

Sharpless NE, Depinho RA. 2006. The mighty mouse: Genetically engineered mouse models in cancer drug development. Nat Rev Drug Discov 5: 741-754.

Stewart TA, Pattengale PK, Leder P. 1984. Spontaneous mammary adenocarcinomas in transgenic mice that carry and express MTV/myc fusion genes. Cell 38: 627-637.

Thomas KR, Capecchi MR. 1987. Site-directed mutagenesis by gene targeting in mouse embryo-derived stem cells. Cell 51: 503-512.

Toogood PL, Harvey PJ, Repine JT, Sheehan DJ, VanderWel SN, Zhou H, Keller PR, McNamara DJ, Sherry D, Zhu T, et al. 2005. Discovery of a potent and selective inhibitor of cyclin-dependent kinase 4/6. J Med Chem 48: 2388-2406.

Warrell RP Jr, He LZ, Richon V, Calleja E, Pandolfi PP. 1998. Therapeutic targeting of transcription in acute promyelocytic leukemia by use of an inhibitor of histone deacetylase. J Natl Cancer Inst 90: 1621- 1625. 


\section{Of Model Pets and Cancer Models: An Introduction to Mouse Models of Cancer}

Andrea Lunardi, Caterina Nardella, John G. Clohessy and Pier Paolo Pandolfi

Cold Spring Harb Protoc; doi: 10.1101/pdb.top069757

\begin{tabular}{cc}
$\begin{array}{r}\text { Email Alerting } \\
\text { Service }\end{array}$ & Receive free email alerts when new articles cite this article - click here. \\
\hline $\begin{array}{r}\text { Subject } \\
\text { Categories }\end{array}$ & $\begin{array}{c}\text { Browse articles on similar topics from Cold Spring Harbor Protocols. } \\
\text { Laboratory Organisms, general (923 articles) } \\
\text { Mouse (437 articles) }\end{array}$ \\
\hline
\end{tabular}

